\title{
Bowel Movement: Integrating Host Mobility and Microbial Transmission Across Host Taxa
}

\author{
Arne Weinhold* \\ Faculty of Biology, Cellular and Organismic Networks, Ludwig-Maximilians-Universität München, Munich, Germany
}

\section{OPEN ACCESS}

Edited by:

Brendan J. M. Bohannan, University of Oregon, United States

Reviewed by: Isabel Gordo, Gulbenkian Institute of Science (IGC),

Portugal

*Correspondence:

Arne Weinhold

arne.weinhold@bio.Imu.de

Specialty section:

This article was submitted to Systems Microbiology,

a section of the journal

Frontiers in Microbiology

Received: 30 November 2021

Accepted: 25 January 2022

Published: 15 February 2022

Citation:

Weinhold A (2022) Bowe Movement: Integrating Host Mobility and Microbial Transmission Across

Host Taxa.

Front. Microbiol. 13:826364. doi: 10.3389/fmicb.2022.826364
The gut microbiota of animals displays a high degree of plasticity with respect to environmental or dietary adaptations and is shaped by factors like social interactions, diet diversity or the local environment. But the contribution of these drivers varies across host taxa and our ability to explain microbiome variability within wild populations remains limited. Terrestrial animals have divergent mobility ranges and can either crawl, walk or fly, from a couple of centimeters toward thousands of kilometers. Animal movement has been little regarded in host microbiota frameworks, though it can directly influence major drivers of the host microbiota: (1) Aggregation movement can enhance social transmissions, (2) foraging movement can extend range of diet diversity, and (3) dispersal movement determines the local environment of a host. Here, I would like to outline how movement behaviors of different host taxa matter for microbial acquisition across mammals, birds as well as insects. Host movement can have contrasting effects and either reduce or enlarge spatial scale. Increased dispersal movement could dissolve local effects of sampling location, while aggregation could enhance inter-host transmissions and uniformity among social groups. Host movement can also extend the boundaries of microbial dispersal limitations and connect habitat patches across plantpollinator networks, while the microbiota of wild populations could converge toward a uniform pattern when mobility is interrupted in captivity or laboratory settings. Hence, the implementation of host movement would be a valuable addition to the metacommunity concept, to comprehend microbial dispersal within and across trophic levels.

Keywords: gut microbiota, movement ecology, microbial dispersal, host movement, migration, environmental acquisition, community assembly, social microbiome

\section{INTRODUCTION}

Microbial associations with animal hosts are ubiquitous and increasingly recognized as important factor for the understanding of host ecology and evolution (Shapira, 2016; Foster et al., 2017; Kolodny et al., 2020; Moeller and Sanders, 2020). The gut microbiota of host animals provides various important roles regarding digestion, provision or nutrients or immune stimulating function within insects as well as vertebrate clades (Moran et al., 2019; Schmidt and Engel, 2021). There is a huge interest in advancing our understanding on how host-associations are formed and how much 
inter-host transmission, environmental acquisition or host genetic factors play a role (Robinson et al., 2019; Mallott and Amato, 2021). While major focus is clearly on functional aspects for the host, reliable transmission routes and acquisition fidelity would sustain microbial associations even in the absence of mutualistic interactions (Leftwich et al., 2020; Sieber et al., 2021). For successful colonization and establishment as resident gut member, microbes must first encounter the host, which is increasingly more likely when one or both of the partners is mobile (Obeng et al., 2021). While microbial motility can be essential for the establishment of symbioses in aquatic and marine environments (Raina et al., 2019), terrestrial animals could directly alter the probability of encountering microbes by relocating themself. Host mobility range and frequency of movements differ across animal taxa, from highly mobile to more resident species.

Various studies applied a metacommunity concept for an ecoevolutionary understanding of host microbiome associations, including environmental microbes as a regional species pool (Adair and Douglas, 2017; Carrier and Reitzel, 2017; Miller et al., 2018). Host movement can extend these theoretical applications from a static toward a more dynamic acquisition process (Mihaljevic, 2012). Host aggregations reduces spatial scale and directly influence probability of inter-host transmissions, while host foraging movements could extend microbial acquisition range or increase microbial dispersal across different habitats.

While insects have (beside vertically transmitted obligate endosymbionts) relatively simple and species poor gut microbiotas (Colman et al., 2012; Engel and Moran, 2013; Jones et al., 2013), most mammals are known for a highly diverse and host taxon specific gut microbiota, that often fits to the phylogenetic distance of the host (the so called "phylosymbiosis" pattern) (Groussin et al., 2017; Groussin et al., 2020; Kohl, 2020). Though this is believed to be the result of an adaptive evolution of mammalian clades for dietary specialization, such a framework does not fit well to the microbiota of birds or bats, which show high inter individual dissimilarity and little influence of host phylogeny (Davenport et al., 2017; Lutz et al., 2019; Song et al., 2020).

With this manuscript I would like to highlight the role of host "movement" and mobility range as a neglected parameter explaining patterns in the microbiota of mammals, birds and insects and the potential transfer across host taxa.

\section{HOW HOST MOVEMENT INCREASES MICROBIAL DISPERSAL}

By moving from one into another place animal hosts can vector microbes over a broad distance and increase their geographic distribution and dispersal rates. At the same time, a host can acquire different microbes from the new location, so that both effects of microbial acquisition and dispersal are often combined and hard to disentangle (Figure 1A). The vinegar fly Drosophila melanogaster is highly attracted by microbial volatiles and use this to find suitable oviposition sites within decaying and rotting fruits (Becher et al., 2012; Markow, 2015; Qiao et al., 2019). The highly

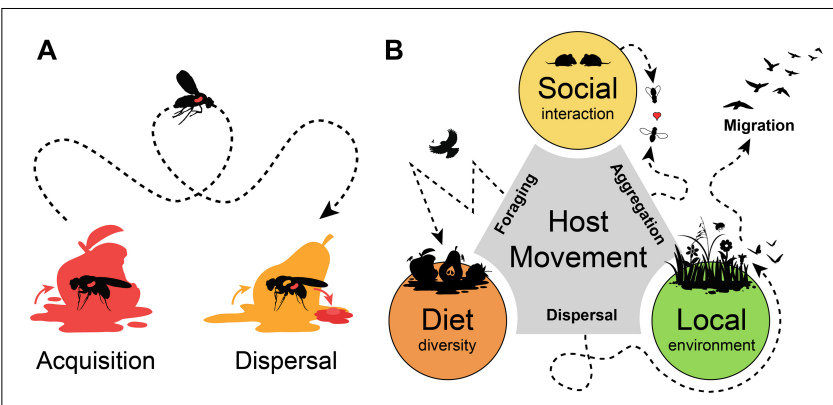

FIGURE 1 | Host movement promotes microbial acquisition and dispersal. (A) Host movement influences microbial acquisition range of a host and promotes microbial dispersal across larger distances. (B) Host movement and mobility range influence major drivers of host microbiota. Host aggregation movement promotes social interactions and microbial transmission among hosts. Foraging movement range determines diet diversity of a host. Dispersal movement, nomadism or migration relocate a host into another location from where environmental microbes can be acquired.

mobile adults can disperse and vector microbes that accelerate the decaying process and support the development of the lowmobile larvae, which is a substantial aspect in the ecology of drosophilid and tephritid fruit flies (Wertheim et al., 2005; Wong et al., 2015; Pais et al., 2018). Under axenic conditions, adult flies respond with a restless behavior and increased locomotion activity (Schretter et al., 2018).

In pollination ecology, the high mobility and movement range of flying insects is a key factor to understand the dispersal abilities of the floral microbiota (Vannette and Fukami, 2017; Morris et al., 2019; Vannette, 2020). Pollinator foraging include repeated visitations of flowers by several different insects species, so that flowers can serve as hubs for microbial exchange within plant pollinator networks (Francis et al., 2021; Keller et al., 2021; Zemenick et al., 2021). Microbes can even directly influence pollinator behavior and preferences, altering floral visitation and nectar removal rates (Schaeffer et al., 2017; Rering et al., 2020; Jacquemyn et al., 2021). Similar, the distribution of multiple pollinator species drives parasite prevalence dynamics over the course of a season (Graystock et al., 2020). Like the common drone fly (Eristalis tenax), which promotes the dispersal of a hymenopteran gut parasite by contaminations of flower tissue with copious defecations (Figueroa et al., 2019; Davis et al., 2021).

\section{HOW HOST MOVEMENT SHAPES THE HOST MICROBIOTA}

A mobile host is not only a spreader, but also a receiver of microbes. Host animals can occupy various environmental niches, so that they can be associated with a diverse set of microbes (Carrier and Reitzel, 2017). The ecology and behavior of the host is an important aspect for host-microbial associations and their movement range would influence probabilities for microbial acquisition from different habitats or during social interaction (Ezenwa et al., 2012; Archie and Tung, 2015; Miller et al., 2018). Animals perform different kinds of movements, 
like non-directional "station-keeping movements" when foraging for food in a restricted area, or "dispersal movement" and "nomadism" as an erratic translocation across different habitats, until "migration" as a highly directional long-distance form of movement (Schlägel et al., 2020). All these movement behaviors could be drivers of the host microbiota, as they allow "social" interactions of a host with conspecifics, influence foraging range and "diet" diversity and determine if a host stays within a single "local" environment or frequently moves across diverse habitats (Figure 1B). Predictions for the outcome could be divergent and case specific, as host mobility could enhance similarities among social group structures within geographic isolated populations. While for other hosts, a high dispersal rate and foraging range could blur local influences and result in high dissimilarities among individuals from a single habitat. Thus, the application of a unified framework for all animal hosts would be challenging, as the influence of stochastic and deterministic factors change with the ecological context of the host and its mobility range (Sieber et al., 2019; Mallott and Amato, 2021).

\section{How Host Movement Defines the Microbial Acquisition Range}

Animals with limited movement abilities depend largely on the regional pool of microbes in their vicinity, which could enhance the correlation of host microbiota with sampling location (Figure 2A, pale yellow). This category includes mainly very small animals or insect larvae. A prominent member would be probably Caenorhabditis elegans, which is literally dwelling in microbial rich habitats as it can be found in nature on decomposing plant material and rotting fruits (Frézal and Félix, 2015). C. elegans is mainly relocated by vectoring animals and its gut microbiota composition is largely determined by the local substrate conditions, which can be distinct from that under laboratory conditions (Zhang et al., 2017). Even larger insect larvae (i.e., caterpillars) are mainly influenced by the foliar microbiota of their local environment, when

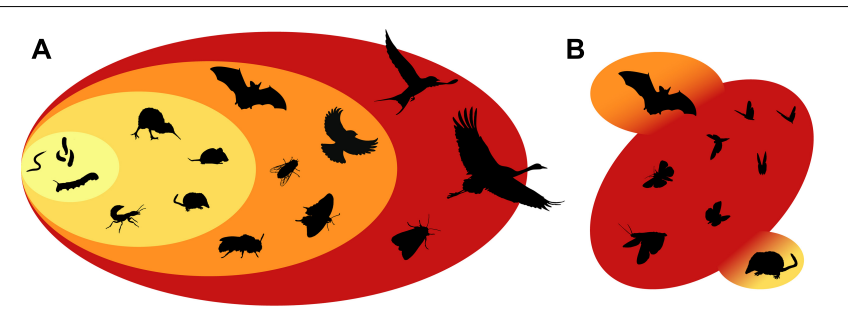

FIGURE 2 | Host movement and mobility ranges across different host taxa. (A) Animals have diverse mobility ranges which could influence the probability for microbial acquisition and dispersal. Small animals or insect larvae (pale yellow) have limited movement abilities, while walking animals (yellow) can move into different environments and interact with other hosts. Flying animals (orange) can extend their foraging range to larger areas, while migrating species (red) can perform long-distance movements. (B) Potential extension of the microbial acquisition range across trophic levels. Insectivorous animals (i.e., bats or shrews) could acquire microbes from their prey (i.e., nocturnal moth) so that their microbiota would resemble more that of flying or migrating species. remaining on a single host plant. Caterpillars are not known to depend on microbial associations nor enrichment of host-specific microbiota and interindividual variability can be explained by different host plants or collection sites (Hammer et al., 2019; Jones et al., 2019; Mason et al., 2020).

The second category contains animals that can perform terrestrial locomotion or "walking" (Figure 2A, yellow). This includes most mammals (including humans), flightless birds or ground-dwelling insects. Such movement abilities increase the mobility range and enable selective foraging for host-specific diets or allows social interactions, which would both increase microbiota similarity among conspecifics. In the wood mouse (Apodemus sylvaticus), the tracking of individual movement patterns revealed, that social connectivity and encounters with conspecifics explains microbiota similarity better than spatial distances or genetic relatedness (Raulo et al., 2021). In humans and primates, social group structures or "co-housing" within a shared living environment have a stronger influence on microbiome similarity among individuals than host-genetic factors or kinship (Tung et al., 2015; Rothschild et al., 2018; Brito et al., 2019; Robinson et al., 2019). Over larger geographic distances dispersal limitations and prey preferences explain gut microbiota dissimilarities among carnivorous mammals in allopatric populations (Moeller et al., 2017). While at a smaller geographic distance $(<25 \mathrm{~km})$, the microbiota of wild mammals does not cluster by location, but mainly by host species identity and dietary preference (Knowles et al., 2019). Movement allows a selective foraging for specific diets within the same habitat, so that herbivorous rodents (mice and voles) are dominated by Bacteroidetes and Firmicutes, while their sympatric insectivorous neighbors (shrews) show increased abundances of Proteobacteria (Knowles et al., 2019).

The third movement category includes all "flying" animals (Figure 2A, orange), which contains most birds, bats as well as several flying insects. Active flight enlarges the range for microbial acquisition further and might dissolve signatures of local habitat boundaries. Characteristic for birds and bats is the low correlation of their microbiota with host phylogeny and a minor influences of sampling locality or habitat type (Hird et al., 2015; Lutz et al., 2019; San Juan et al., 2020; Bodawatta et al., 2021). Studies that have combined individual flight behavior of birds with host microbiota analysis are extremely rare, but for the barn owl (Tyto alba) the medium foraging range indicated a positive correlation with microbial diversity (Corl et al., 2020). Also insects showed typically high dissimilarities among individuals, low biogeographic patterns and only a weak influence of host phylogeny (Colman et al., 2012; Jones et al., 2013; Yun et al., 2014; Bahrndorff et al., 2017; Wang et al., 2020). But flying insects do not necessarily cover larger absolute geographic distances than terrestrial animals, but mobility per se with a high frequency of movements within a smaller range could expose a host to heterogeneous microenvironments. This is particularly important for microbe-plant-pollinator interactions, since the dispersal of nectar microbes is directly linked to the foraging behavior of pollinators (Cullen et al., 2021; Francis et al., 2021). Wild bee species vary in their foraging ranges from a few hundred meters to several kilometers, while nocturnal 
moth can easily exceed movement ranges to several hundred kilometers (Greenleaf et al., 2007; Satterfield et al., 2020).

The fourth movement category (Figure 2A, red) includes species that "migrate," which differs from the previous movement categories, as it is a seasonal directional movement over larger geographic distance. Migration has been primarily investigated in birds and butterflies regarding the long-distance dispersal of parasites or as a strategy to avoid pathogen infections (Altizer et al., 2011; Bartel et al., 2011; Boulinier et al., 2016; Viana et al., 2016). But a few studies have investigated if host migration influences gut microbial diversity. While migrating passerine birds showed a change of their microbiota following a dietary shift (Lewis et al., 2017; Skeen et al., 2021), other studies pointed mainly at physiological adaptations and little environmental acquisition of microbes (Risely et al., 2017, 2018; Wu et al., 2018; Turjeman et al., 2020). The influence of migration on the microbiota of birds seems rather marginal and could be mainly attributed to dietary shifts between geographic distant locations. With insects, the influence of migration is less clear. Though, insect migration is often associated with the spectacular mass migration events of the monarch butterfly (Danaus plexippus) or the painted lady (Vanessa cardui), migration abilities are not uncommon for other butterflies or nocturnal moth, but remain often unnoticed as they fly in lower numbers or at night (Chowdhury et al., 2021). But even seasonal mass migration events are easily overlooked, when insects are small and fly at high altitudes like the marmalade hoverfly (Episyrphus balteatus) (Hu et al., 2016; Wotton et al., 2019; Satterfield et al., 2020). But to what extent insect migration would influence microbiota acquisition or dispersal has not been fully elucidated yet.

\section{Does 'Flight' or the Consumption of Mobile Insect Prey Shape the Host Microbiota?}

A recent comparative study concludes that a convergent physiological adaption to "flight" (reduced dependence on microbes to reduce weight of the digestive system) shapes the bird as well as the bat microbiota (Song et al., 2020). Particular Proteobacteria seem somehow associated with "flight," as they are commonly found in birds, bats and insects, while most mammals are dominated by Bacteroidetes and Firmicutes (Brooks et al., 2016; Song et al., 2020). But there are notable exceptions. In the analysis of Song et al. (2020) the order of Insectivora (here mainly shrews of the genus Crocidura spp.) showed the second highest proportions of Proteobacteria and a less mammalspecific microbiota than Chiroptera (bats), directly followed by Pholidota (ant-eating Pangolins). If the observed pattern would be explained by a physiological adaptation to flight, this raises the question why terrestrial shrews have the most "bird-like" microbiota of all mammals.

What is intriguing about this observation is the possibility that insectivorous mammals might obtain their microbiota directly from their prey. In such a scenario, the consumption of insects would expand their microbial acquisition range, which resembles more that of a flying insect (Figure 2B). A transfer of microbes across predator-prey networks has been suggested for insectivorous birds and predatory insects (Tiede et al., 2017; Suenami et al., 2019; Dion-Phénix et al., 2021). The mammalian microbiota is strongly influenced by species identity and type of diet, but an increase of invertebrate prey (i.e., insects) within the diet correlates with a decrease in bacterial alpha diversity compared to mammals with a primarily herbivorous lifestyle (Knowles et al., 2019; Harrison et al., 2021). Tough "insectivory" includes the consumption of non-flying insects (i.e., ants or termites) as well as other invertebrates, such a dietary preference seems to result in a convergent adaptation in the microbiota of phylogenetically distant mammalian clades (Pilosa, Cingulata, Tubulidentata, and Carnivora) (Delsuc et al., 2014). Particular bats might be able to further expand their microbial acquisition range beyond their own flight range, as they consume a diverse variety of highly mobile insect species within the orders Lepidoptera, Diptera, and Coleoptera (Tiede et al., 2020). The Brazilian free-tailed bat (Tadarida brasiliensis) preys on high altitude flying insects, which includes several migrating species, and noctuid moth make on average $77 \%$ of their diet (Krauel et al., 2018). There is clearly more work needed to clarify to what extend the microbiota of insects influences the microbiota of insectivorous animals across tropic levels (Figure 2B), and what patterns would be predicted for the microbiota of highly mobile hosts.

\section{Interruption of Host Movement Behavior in Captivity or Laboratory Settings}

Captivity and laboratory settings can alter the outcome of microbiome studies tremendously and should be taken with caution when implying evolutionary context (Hird, 2017). As long as the insectivorous bat Mops condylurus preys on flying insects, they show higher interindividual variability with relative low alpha diversity in their fecal microbiota, but converge toward a more uniform community composition with increased alpha diversity when kept in captivity for 6 weeks (Edenborough et al., 2020). When brought into captivity, primates tend to shift toward a more "human-like" microbiota (Nishida and Ochman, 2021), which was attributed to a reduced diversity of food plants with lower fiber content compared to the naturally foraged diet of wild relatives (Clayton et al., 2016). Captivity has a significant effect on the microbiota of several mammal species (Kohl et al., 2014; McKenzie et al., 2017), and there is a lot potential to further explore how restrictions in foraging movement and range size shape the microbiome. Captivity alters also the microbiota of migratory as well as terrestrial birds, such as the crane and the brown kiwi (Xie et al., 2016; San Juan et al., 2021). Any restriction of animal movement behavior could disrupt natural host-microbial dispersal routes leading to more "uniform" results that confound the outcome obtained in laboratory settings or under captivity conditions.

\section{CONCLUSION AND FUTURE PERSPECTIVES}

A major challenge in the investigation of the microbiota of wild animals is the lack of a clear framework of what can be expected 

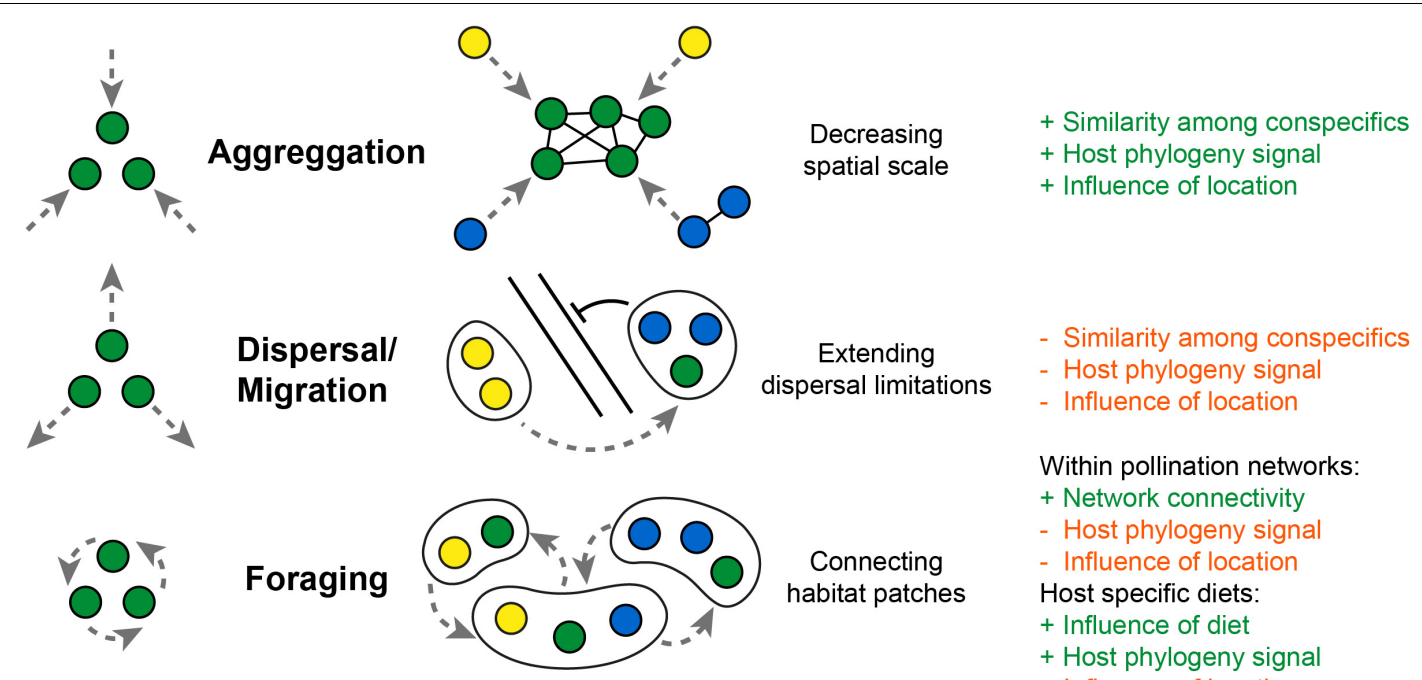

Connecting habitat patches
Within pollination networks:

+ Network connectivity

- Host phylogeny signal

- Influence of location

Host specific diets:

+ Influence of diet

+ Host phylogeny signal

- Influence of location

FIGURE 3 | Potential framework how moving hosts (as "floating islands") influence parameter of microbiota composition. Aggregation movement decreases spatial scale and promotes social exchange among conspecifics, which could increase correlation by host phylogeny. Dispersal movement could extend dispersal limitations of microbes but weakens the influence of local landscape parameters or host phylogeny. Foraging movement could connect habitat patches by distributing microbes across plant-pollinator networks and weakens influences of host phylogeny or the local environment. While a selective foraging for host specific diets (e.g., mammalian feeding guilds) would increase correlations with host diet and phylogeny (+ and - refers to an increase or decrease in correlation).

when host species identity or geographic location are failing short to predict the observed variability within wild populations. By an integration of flight behavior, the study by Song et al. (2020) marks a transition that elevates from a pure host phylogenetic perspective toward the integration of host ecology. There is an exciting potential for future research to combine hypothesis about animal behavior and movement decisions with the host microbiota (Davidson et al., 2020; Bo and Kohl, 2021).

In the past, the analysis of host microbiomes has been mainly performed from a "host-centric" viewpoint, following the tradition of genetic model systems in the search of a host-genetic basis that explains microbiome composition. The inclusion of the "microbes perspective" has challenged this view and brings community ecological principles and stochastic processes into host microbiota analysis (Obeng et al., 2021; Sieber et al., 2021). The host microbiota is not a constant trait, but shows context dependent plasticity and interindividual variability in time and space (Carrier and Reitzel, 2017). Especially the metacommunity concept has become very useful in describing host-associated microbiota as it provides a framework that integrates interhost transmission and environmental acquisition of microbes from an external pool (Miller et al., 2018). As a reference to island biogeography, hosts are often depicted as passive "microbial habitats" that become colonized by microbes, similar as islands become colonized by other macrobiota, while social connectivity is illustrated by clustering single host "islands" into "archipelagoes" as used in the analogy by Sarkar et al. (2020). Though this mainly refers to stable social group structures, it misses to depict the dynamic and transient nature of these interactions. Moving hosts would be more comparable to "floating islands" which constantly change their spatial distribution relative to each other, actively connect in social interactions or relocate themselves into different environments (Figure 3).

Similar as microbial movement (motility) turned out to be an important aspect of host microbiota associations within aquatic systems (Raina et al., 2019), host movement (mobility) could become a vital addition to the metacommunity concept explaining the acquisition and dispersal of microbes among terrestrial animals. Movement changes several parameters that influence microbiome composition and could strength similarities among conspecifics via selective foraging or social exchange (Figure 3). Social transmission would become merely a consequence of aggregation movement, which decreases spatial scale and enhances the probability of microbial transmission among conspecifics. But movement could also blur correlations with host phylogeny and lead to higher dissimilarity among conspecifics from the same location. The mobility range and foraging pattern of a host could be a key factor to fully comprehend the composition and diversity of the host microbiota. Though a direct tracking of host movement in the wild is indeed a challenge, the outstanding work by Raulo et al. (2021) and Skeen et al. (2021) demonstrates the value of repeated sampling and the integration of movement patterns as a new dimension into microbiota analysis of birds and mammals. But even for insects, where a direct tracking of individuals is less feasible, the investigation of transmission routes within plant pollinator networks becomes a promising step to better understand microbial dispersal among different host taxa and across the animal and plant kingdom (Keller et al., 2021; Zemenick et al., 2021). Still, a framework for predictions of microbiota composition of highly mobile flying hosts is missing, as increasing complexity of interactions makes it difficult to directly correlate host mobility range with gut microbial 
diversity. Here, more work is needed to further explore if the same drivers can explain microbiota composition of flying hosts, resolving the patterns observed from insects, birds and bats.

\section{DATA AVAILABILITY STATEMENT}

The original contributions presented in the study are included in the article/supplementary material, further inquiries can be directed to the corresponding author/s.

\section{REFERENCES}

Adair, K. L., and Douglas, A. E. (2017). Making a microbiome: the many determinants of host-associated microbial community composition. Curr. Opin. Microbiol. 35, 23-29. doi: 10.1016/j.mib.2016.11.002

Altizer, S., Bartel, R., and Han, B. A. (2011). Animal migration and infectious disease risk. Science 331, 296-302. doi: 10.1126/science.1194694

Archie, E. A., and Tung, J. (2015). Social behavior and the microbiome. Curr. Opin. Behav. Sci. 6, 28-34. doi: 10.1016/j.cobeha.2015.07.008

Bahrndorff, S., Jonge, N., De, Skovgård, H., and Nielsen, J. L. (2017). Bacterial communities associated with houseflies (Musca domestica L.) sampled within and between farms. PLoS One 12:e0169753. doi: 10.1371/journal.pone.0169753

Bartel, R. A., Oberhauser, K. S., Roode, J. C., De, and Altizer, S. M. (2011). Monarch butterfly migration and parasite transmission in eastern North America. Ecology 92, 342-351. doi: 10.1890/10-0489.1

Becher, P. G., Flick, G., Rozpedowska, E., Schmidt, A., and Hagman, A. (2012). Yeast, not fruit volatiles mediate Drosophila melanogaster attraction, oviposition and development. Funct. Ecol. 26, 822-828. doi: 10.1111/j.13652435.2012.02006.x

Bo, T., and Kohl, K. D. (2021). Stabilization and optimization of host-microbeenvironment interactions as a potential reason for the behavior of natal philopatry. Anim. Microbiome. 3:26. doi: 10.1186/s42523-021-00087-3

Bodawatta, K. H., Hird, S. M., Grond, K., Poulsen, M., and Jønsson, K. A. (2021). Avian gut microbiomes taking flight. Trends Microbiol. [Epub online ahead of print] doi: 10.1016/j.tim.2021.07.003

Boulinier, T., Kada, S., Ponchon, A., Dupraz, M., Dietrich, M., Gamble, A., et al. (2016). Migration, Prospecting, Dispersal? What Host Movement Matters for Infectious Agent Circulation? Integr. Comp. Biol. 56, 330-342. doi: 10.1093/icb/ icw015

Brito, I. L., Gurry, T., Zhao, S., Huang, K., Young, S. K., Shea, T. P., et al. (2019). Transmission of human-associated microbiota along family and social networks. Nat. Microbiol. 4, 964-971. doi: 10.1038/s41564-019-0409-6

Brooks, A. W., Kohl, K. D., Brucker, R. M., Opstal, E. J., van, and Bordenstein, S. R. (2016). Phylosymbiosis: relationships and Functional Effects of Microbial Communities across Host Evolutionary History. PLoS Biol. 14:e2000225. doi: 10.1371/journal.pbio.2000225

Carrier, T. J., and Reitzel, A. M. (2017). The hologenome across environments and the implications of a host-associated microbial repertoire. Front. Microbiol. 8:802. doi: $10.3389 /$ fmicb.2017.00802

Chowdhury, S., Fuller, R. A., Dingle, H., Chapman, J. W., and Zalucki, M. P. (2021). Migration in butterflies: a global overview. Biol. Rev. 96, 1462-1483. doi: 10.1111/brv.12714

Clayton, J. B., Vangay, P., and Huang, H. (2016). Captivity humanizes the primate microbiome. Proc. Natl. Acad. Sci. U.S.A. 113, 10376-10381. doi: 10.1073/pnas. 1521835113

Colman, D. R., Toolson, E. C., and Takacs-Vesbach, C. D. (2012). Do diet and taxonomy influence insect gut bacterial communities? Mol. Ecol. 21, 5124-5137. doi: 10.1111/j.1365-294X.2012.05752.x

Corl, A., Charter, M., Rozman, G., Toledo, S., Turjeman, S., Kamath, P. L., et al. (2020). Movement ecology and sex are linked to barn owl microbial community composition. Mol. Ecol. 29, 1358-1371. doi: 10.1111/mec.15398

Cullen, N. P., Fetters, A. M., and Ashman, T. L. (2021). Integrating microbes into pollination. Curr. Opin. Insect Sci. 44, 48-54. doi: 10.1016/j.cois.2020. 11.002

\section{AUTHOR CONTRIBUTIONS}

AW conceived, designed, and wrote the manuscript.

\section{ACKNOWLEDGMENTS}

I would like to thank the reviewer and the editor for their constructive comments and all authors and scientists that dedicated their work on PhyloPic to the public domain.

Davenport, E. R., Sanders, J. G., Song, S. J., Amato, K. R., Clark, A. G., and Knight, R. (2017). The human microbiome in evolution. BMC Biol. 15:127. doi: 10.1186/s12915-017-0454-7

Davidson, G. L., Raulo, A., and Knowles, S. C. L. (2020). Identifying MicrobiomeMediated Behaviour in Wild Vertebrates. Trends Ecol. Evol. 35, 972-980. doi: 10.1016/j.tree.2020.06.014

Davis, A. E., Deutsch, K. R., and Torres, A. M. (2021). Eristalis flower flies can be mechanical vectors of the common trypanosome bee parasite. Crithidia Bombi. Sci. Rep. 11:15852. doi: 10.1038/s41598-021-95323-w

Delsuc, F., Metcalf, J. L., Wegener Parfrey, L., Song, S. J., González, A., and Knight, R. (2014). Convergence of gut microbiomes in myrmecophagous mammals. Mol. Ecol. 23, 1301-1317. doi: 10.1111/mec.12501

Dion-Phénix, H., Charmantier, A., Franceschi, C., de, Bourret, G., Kembel, S. W., et al. (2021). Bacterial microbiota similarity between predators and prey in a blue tit trophic network. ISME J. 15, 1098-1107. doi: 10.1038/s41396-02000836-3

Edenborough, K. M., Mu, A., Mühldorfer, K., Lechner, J., Lander, A., Bokelmann, M., et al. (2020). Microbiomes in the insectivorous bat species Mops condylurus rapidly converge in captivity. PLoS One 15:e223629. doi: 10.1371/journal.pone. 0223629

Engel, P., and Moran, N. A. (2013). The gut microbiota of insects - diversity in structure and function. FEMS Microbiol. Rev. 37, 699-735. doi: 10.1111/15746976.12025

Ezenwa, V. O., Gerardo, N. M., Inouye, D. W., Medina, M., and Xavier, J. B. (2012). Animal behavior and the microbiome. Science 338, 198-199. doi: 10. $1126 /$ science. 1227412

Figueroa, L. L., Blinder, M., and Grincavitch, C. (2019). Bee pathogen transmission dynamics: deposition, persistence and acquisition on flowers. Proc. R. Soc. B Biol. Sci. 286:20190603. doi: 10.1098/rspb.2019.0603

Foster, K. R., Schluter, J., Coyte, K. Z., and Rakoff-Nahoum, S. (2017). The evolution of the host microbiome as an ecosystem on a leash. Nature 548, 43-51. doi: $10.1038 /$ nature23292

Francis, J. S., Tatarko, A. R., Richman, S. K., Vaudo, A. D., and Leonard, A. S. (2021). Microbes and pollinator behavior in the floral marketplace. Curr. Opin. Insect Sci. 44, 16-22. doi: 10.1016/j.cois.2020.10.003

Frézal, L., and Félix, M. A. (2015). C. elegans outside the Petri dish. Elife 4:e05849. doi: 10.7554/eLife.05849

Graystock, P., Ng, W. H., Parks, K., Tripodi, A. D., Muñiz, P. A., Fersch, A. A., et al. (2020). Dominant bee species and floral abundance drive parasite temporal dynamics in plant-pollinator communities. Nat. Ecol. Evol. 4, 1358-1367. doi: 10.1038/s41559-020-1247-x

Greenleaf, S. S., Williams, N. M., Winfree, R., and Kremen, C. (2007). Bee foraging ranges and their relationship to body size. Oecologia 153, 589-596. doi: 10.1007/ s00442-007-0752-9

Groussin, M., Mazel, F., and Alm, E. J. (2020). Co-evolution and Co-speciation of Host-Gut Bacteria Systems. Cell Host Microbe 28, 12-22. doi: 10.1016/j.chom. 2020.06.013

Groussin, M., Mazel, F., Sanders, J. G., Smillie, C. S., Lavergne, S., Thuiller, W., et al. (2017). Unraveling the processes shaping mammalian gut microbiomes over evolutionary time. Nat. Commun. 8:14319. doi: 10.1038/ncomms14319

Hammer, T. J., Sanders, J. G., and Fierer, N. (2019). Not all animals need a microbiome. FEMS Microbiol. Lett. 366:fnz117. doi: 10.1093/femsle/fnz117

Harrison, X. A., McDevitt, A. D., and Dunn, J. C. (2021). Fungal microbiomes are determined by host phylogeny and exhibit widespread associations with the 
bacterial microbiome. Proc. R. Soc. B Biol. Sci. 288:20210552. doi: 10.1098/rspb. 2021.0552

Hird, S. M. (2017). Evolutionary Biology Needs Wild Microbiomes. Front. Microbiol. 8:725. doi: 10.3389/fmicb.2017.00725

Hird, S. M., Sánchez, C., Carstens, B. C., and Brumfield, R. T. (2015). Comparative Gut Microbiota of 59 Neotropical Bird Species. Front. Microbiol. 6:1403. doi: 10.3389/fmicb.2015.01403

Hu, G., Lim, K. S., Horvitz, N., Clark, S. J., Reynolds, D. R., Sapir, N., et al. (2016). Mass seasonal bioflows of high-flying insect migrants. Science 354, 1584-1587. doi: 10.1126/science.aah4379

Jacquemyn, H., Pozo, M. I., Álvarez-Pérez, S., Lievens, B., and Fukami, T. (2021). Yeast-nectar interactions: metacommunities and effects on pollinators. Curr. Opin. Insect Sci. 44, 35-40. doi: 10.1016/j.cois.2020.09.014

Jones, A. G., Mason, C. J., Felton, G. W., and Hoover, K. (2019). Host plant and population source drive diversity of microbial gut communities in two polyphagous insects. Sci. Rep. 9:2792. doi: 10.1038/s41598-019-39163-9

Jones, R. T., Sanchez, L. G., and Fierer, N. (2013). A Cross-Taxon Analysis of InsectAssociated Bacterial Diversity. PLoS One 8:e61218. doi: 10.1371/journal.pone. 0061218

Keller, A., McFrederick, Q. S., Dharampal, P., Steffan, S., Danforth, B. N., and Leonhardt, S. D. (2021). (More than) Hitchhikers through the network: the shared microbiome of bees and flowers. Curr. Opin. Insect Sci. 44, 8-15. doi: 10.1016/j.cois.2020.09.007

Knowles, S. C. L., Eccles, R. M., and Baltrūnaite, L. (2019). Species identity dominates over environment in shaping the microbiota of small mammals. Ecol. Lett. 22, 826-837. doi: 10.1111/ele.13240

Kohl, K. D. (2020). Ecological and evolutionary mechanisms underlying patterns of phylosymbiosis in host-associated microbial communities. Philos. Trans. R. Soc. B Biol. Sci. 375:20190251 doi: 10.1098/rstb.2019.0251

Kohl, K. D., Skopec, M. M., and Dearing, M. D. (2014). Captivity results in disparate loss of gut microbial diversity in closely related hosts. Conserv. Physiol. 2:cou009. doi: 10.1093/conphys/cou009

Kolodny, O., Callahan, B. J., and Douglas, A. E. (2020). The role of the microbiome in host evolution. Philos. Trans. R. Soc. Lond. B. Biol. Sci. 375:20190588. doi: 10.1098/rstb.2019.0588

Krauel, J. J., Brown, V. A., Westbrook, J. K., and McCracken, G. F. (2018). Predator-prey interaction reveals local effects of high-altitude insect migration. Oecologia 186, 49-58. doi: 10.1007/s00442-017-3995-0

Leftwich, P. T., Edgington, M. P., and Chapman, T. (2020). Transmission efficiency drives host-microbe associations. Proc. R. Soc. B Biol. Sci. 287:20200820. doi: 10.1098/rspb. 2020.0820

Lewis, W. B., Moore, F. R., and Wang, S. (2017). Changes in gut microbiota of migratory passerines during stopover after crossing an ecological barrier. Auk 134, 137-145. doi: 10.1642/AUK-16-120.1

Lutz, H. L., Jackson, E. W., Webala, P. W., Babyesiza, W. S., Kerbis Peterhans, J. C., Demos, T. C., et al. (2019). Ecology and Host Identity Outweigh Evolutionary History in Shaping the Bat Microbiome. mSystems 4, e511-e519. doi: 10.1128/ mSystems.00511-19

Mallott, E. K., and Amato, K. R. (2021). Host specificity of the gut microbiome. Nat. Rev. Microbiol. 19, 639-653. doi: 10.1038/s41579-021-00562-3

Markow, T. A. (2015). The secret lives of Drosophila flies. Elife 4:e06793. doi: $10.7554 /$ eLife.06793

Mason, C. J., St Clair, A., and Peiffer, M. (2020). Diet influences proliferation and stability of gut bacterial populations in herbivorous lepidopteran larvae Y. Lou, ed. PLoS One 15:e0229848. doi: 10.1371/journal.pone.0229848

McKenzie, V. J., Song, S. J., and Delsuc, F. (2017). The effects of captivity on the mammalian gut microbiome. Integr. Comp. Biol. 57, 690-704. doi: 10.1093/icb/ icx090

Mihaljevic, J. R. (2012). Linking metacommunity theory and symbiont evolutionary ecology. Trends Ecol. Evol. 27, 323-329. doi: 10.1016/j.tree.2012. 01.011

Miller, E. T., Svanbäck, R., and Bohannan, B. J. M. (2018). Microbiomes as Metacommunities: understanding Host-Associated Microbes through Metacommunity Ecology. Trends Ecol. Evol. 33, 926-935. doi: 10.1016/j.tree. 2018.09.002

Moeller, A. H., and Sanders, J. G. (2020). Roles of the gut microbiota in the adaptive evolution of mammalian species: microbiotas alter mammalian adaptation. Philos. Trans. R. Soc. B Biol. Sci. 375:20190597. doi: 10.1098/rstb.2019.0597
Moeller, A. H., Suzuki, T. A., Lin, D., Lacey, E. A., Wasser, S. K., and Nachman, M. W. (2017). Dispersal limitation promotes the diversification of the mammalian gut microbiota. Proc. Natl. Acad. Sci. U.S.A. 114, 13768-13773. doi: $10.1073 /$ pnas. 1700122114

Moran, N. A., Ochman, H., and Hammer, T. J. (2019). Evolutionary and Ecological Consequences of Gut Microbial Communities. Annu. Rev. Ecol. Evol. Syst. 50, 451-475. doi: 10.1146/annurev-ecolsys-110617-062453

Morris, M. M., Frixione, N. J., Burkert, A. C., Dinsdale, E. A., and Vannette, R. L. (2019). Microbial abundance, composition, and function in nectar are shaped by flower visitor identity. FEMS Microbiol. Ecol. 96:fiaa003. doi: 10.1093/ femsec/fiaa003

Nishida, A. H., and Ochman, H. (2021). Captivity and the co-diversification of great ape microbiomes. Nat. Commun. 12:5632. doi: 10.1038/s41467-02125732-y

Obeng, N., Bansept, F., Sieber, M., Traulsen, A., and Schulenburg, H. (2021). Evolution of Microbiota-Host Associations: the Microbe's Perspective. Trends Microbiol. 29, 779-787. doi: 10.1016/j.tim.2021.02.005

Pais, I. S., Valente, R. S., Sporniak, M., and Teixeira, L. (2018). Drosophila melanogaster establishes a species-specific mutualistic interaction with stable gut-colonizing bacteria. PLoS Biol. 16:e2005710. doi: 10.1371/journal.pbio. 2005710

Qiao, H., Keesey, I. W., Hansson, B. S., and Knaden, M. (2019). Gut microbiota affects development and olfactory behavior in Drosophila melanogaster. J. Exp. Biol. 222:jeb192500. doi: 10.1242/jeb.192500

Raina, J. B., Fernandez, V., Lambert, B., Stocker, R., and Seymour, J. R. (2019). The role of microbial motility and chemotaxis in symbiosis. Nat. Rev. Microbiol. 17, 284-294. doi: 10.1038/s41579-019-0182-9

Raulo, A., Allen, B. E., Troitsky, T., Husby, A., Firth, J. A., Coulson, T., et al. (2021). Social networks strongly predict the gut microbiota of wild mice. ISME J. 15, 2601-2613. doi: 10.1038/s41396-021-00949-3

Rering, C. C., Vannette, R. L., Schaeffer, R. N., and Beck, J. J. (2020). Microbial Co-Occurrence in Floral Nectar Affects Metabolites and Attractiveness to a Generalist Pollinator. J. Chem. Ecol. 46, 659-667. doi: 10.1007/s10886-02001169-3

Risely, A., Waite, D., Ujvari, B., Klaassen, M., and Hoye, B. (2017). Gut microbiota of a long-distance migrant demonstrates resistance against environmental microbe incursions. Mol. Ecol. 26, 5842-5854. doi: 10.1111/mec.1 4326

Risely, A., Waite, D. W., Ujvari, B., Hoye, B. J., and Klaassen, M. (2018). Active migration is associated with specific and consistent changes to gut microbiota in Calidris shorebirds. J. Anim. Ecol. 87, 428-437. doi: 10.1111/1365-2656. 12784

Robinson, C. D., Bohannan, B. J., and Britton, R. A. (2019). Scales of persistence: transmission and the microbiome. Curr. Opin. Microbiol. 50, 42-49. doi: 10. 1016/j.mib.2019.09.009

Rothschild, D., Weissbrod, O., and Barkan, E. (2018). Environment dominates over host genetics in shaping human gut microbiota. Nature 555, 210-215. doi: $10.1038 /$ nature25973

San Juan, P. A., Castro, I., and Dhami, M. K. (2021). Captivity reduces diversity and shifts composition of the Brown Kiwi microbiome. Anim. Microbiome 3:48. doi: 10.1186/s42523-021-00109-0

San Juan, P. A., Hendershot, J. N., Daily, G. C., and Fukami, T. (2020). Landuse change has host-specific influences on avian gut microbiomes. ISME J. 14, 318-321. doi: 10.1038/s41396-019-0535-4

Sarkar, A., Harty, S., and Johnson, K. V. A. (2020). Microbial transmission in animal social networks and the social microbiome. Nat. Ecol. Evol. 4, 10201035. doi: 10.1038/s41559-020-1220-8

Satterfield, D. A., Sillett, T. S., Chapman, J. W., Altizer, S., and Marra, P. P. (2020). Seasonal insect migrations: massive, influential, and overlooked. Front. Ecol. Environ. 18:335-344. doi: 10.1002/fee.2217

Schaeffer, R. N., Mei, Y. Z., Andicoechea, J., Manson, J. S., and Irwin, R. E. (2017) Consequences of a nectar yeast for pollinator preference and performance. Funct. Ecol. 31, 613-621. doi: 10.1111/1365-2435.12762

Schlägel, U. E., Grimm, V., and Blaum, N. (2020). Movement-mediated community assembly and coexistence. Biol. Rev. 95, 1073-1096. doi: 10.1111/ brv. 12600

Schmidt, K., and Engel, P. (2021). Mechanisms underlying gut microbiota-host interactions in insects. J. Exp. Biol. 224:jeb207696 doi: 10.1242/jeb.207696 
Schretter, C. E., Vielmetter, J., Bartos, I., Marka, Z., Marka, S., Argade, S., et al. (2018). A gut microbial factor modulates locomotor behaviour in Drosophila. Nature 563, 402-406. doi: 10.1038/s41586-018-0634-9

Shapira, M. (2016). Gut Microbiotas and Host Evolution: scaling Up Symbiosis. Trends Ecol. Evol. 31, 539-549. doi: 10.1016/j.tree.2016.03.006

Sieber, M., Pita, L., and Weiland-Bräuer, N. (2019). Neutrality in the metaorganism. PLoS Biol. 17:e3000298. doi: 10.1371/journal.pbio.3000298

Sieber, M., Traulsen, A., Schulenburg, H., and Douglas, A. E. (2021). On the evolutionary origins of host-microbe associations. Proc. Natl. Acad. Sci. U.S.A. 118.e2016487118 doi: 10.1073/pnas.2016487118

Skeen, H. R., Cooper, N. W., Hackett, S. J., Bates, J. M., and Marra, P. P. (2021). Repeated sampling of individuals reveals impact of tropical and temperate habitats on microbiota of a migratory bird. Mol. Ecol. 30, 5900-5916. doi: $10.1111 / \mathrm{mec} .16170$

Song, S. J., Sanders, J. G., and Delsuc, F. (2020). Comparative Analyses of Vertebrate Gut Microbiomes Reveal Convergence between Birds and Bats. MBio 11, e02901-19. doi: 10.1128/mBio.02901-19

Suenami, S., Konishi Nobu, M., and Miyazaki, R. (2019). Community analysis of gut microbiota in hornets, the largest eusocial wasps, Vespa mandarinia and V. simillima. Sci. Rep. 9:9830. doi: 10.1038/s41598-019-46388-1

Tiede, J., Diepenbruck, M., Gadau, J., Wemheuer, B., Daniel, R., and Scherber, C. (2020). Seasonal variation in the diet of the serotine bat (Eptesicus serotinus): a high-resolution analysis using DNA metabarcoding. Basic Appl. Ecol. 49, 1-12. doi: 10.1016/j.baae.2020.09.004

Tiede, J., Scherber, C., Mutschler, J., McMahon, K. D., and Gratton, C. (2017). Gut microbiomes of mobile predators vary with landscape context and species identity. Ecol. Evol. 7, 8545-8557. doi: 10.1002/ece3.3390

Tung, J., Barreiro, L. B., and Burns, M. B. (2015). Social networks predict gut microbiome composition in wild baboons. Elife 4:e05224. doi: 10.7554/eLife. 05224

Turjeman, S., Corl, A., and Wolfenden, A. (2020). Migration, pathogens and the avian microbiome: a comparative study in sympatric migrants and residents. Mol. Ecol. 29, 4706-4720. doi: 10.1111/mec.15660

Vannette, R. L. (2020). The Floral Microbiome: plant, Pollinator, and Microbial Perspectives. Annu. Rev. Ecol. Evol. Syst. 51, 363-386. doi: 10.1146/annurevecolsys-011720-013401

Vannette, R. L., and Fukami, T. (2017). Dispersal enhances beta diversity in nectar microbes. Ecol. Lett. 20, 901-910. doi: 10.1111/ele.12787

Viana, D. S., Santamaría, L., and Figuerola, J. (2016). Migratory Birds as Global Dispersal Vectors. Trends Ecol. Evol. 31, 763-775. doi: 10.1016/j.tree.2016.0 7.005

Wang, Y., Kapun, M., Waidele, L., Kuenzel, S., Bergland, A. O., and Staubach, F. (2020). Common structuring principles of the Drosophila melanogaster microbiome on a continental scale and between host and substrate. Environ. Microbiol. Rep. 12, 220-228. doi: 10.1111/1758-2229.12826
Wertheim, B., Baalen, E. J. A., Van, Dicke, M., and Vet, L. E. M. (2005). Pheromonemediated aggregation in non-social arthropods: an evolutionary ecological perspective. Annu. Rev. Entomol. 50, 321-346. doi: 10.1146/annurev.ento.49. 061802.123329

Wong, A. C. N., Luo, Y., Jing, X., Franzenburg, S., Bost, A., and Douglas, A. E. (2015). The host as the driver of the microbiota in the gut and external environment of Drosophila melanogaster. Appl. Environ. Microbiol. 81, 62326240. doi: 10.1128/AEM.01442-15

Wotton, K. R., Gao, B., Menz, M. H. M., Morris, R. K. A., Ball, S. G., Lim, K. S., et al. (2019). Mass Seasonal Migrations of Hoverflies Provide Extensive Pollination and Crop Protection Services. Curr. Biol. 29:2167-2173.e5. doi: 10.1016/j.cub. 2019.05.036

Wu, Y., Yang, Y., Cao, L., Yin, H., Xu, M., Wang, Z., et al. (2018). Habitat environments impacted the gut microbiome of long-distance migratory swan geese but central species conserved. Sci. Rep. 8:13314. doi: 10.1038/s41598-01831731-9

Xie, Y., Xia, P., Wang, H., Yu, H., Giesy, J. P., Zhang, Y., et al. (2016). Effects of captivity and artificial breeding on microbiota in feces of the red-crowned crane (Grus japonensis). Sci. Rep. 6:33350. doi: 10.1038/srep33350

Yun, J. H., Roh, S. W., and Whon, T. W. (2014). Insect gut bacterial diversity determined by environmental habitat, diet, developmental stage, and phylogeny of host. Appl. Environ. Microbiol. 80, 5254-5264. doi: 10.1128/AEM.01226-14

Zemenick, A. T., Vanette, R. L., and Rosenheim, J. A. (2021). Linked networks reveal dual roles of insect dispersal and species sorting for bacterial communities in flowers. Oikos 130, 697-707. doi: 10.1111/oik.06818

Zhang, F., Berg, M., Dierking, K., Félix, M. A., Shapira, M., Samuel, B. S., et al. (2017). Caenorhabditis elegans as a model for microbiome research. Front. Microbiol. 8:485. doi: 10.3389/fmicb.2017.00485

Conflict of Interest: The author declares that the research was conducted in the absence of any commercial or financial relationships that could be construed as a potential conflict of interest.

Publisher's Note: All claims expressed in this article are solely those of the authors and do not necessarily represent those of their affiliated organizations, or those of the publisher, the editors and the reviewers. Any product that may be evaluated in this article, or claim that may be made by its manufacturer, is not guaranteed or endorsed by the publisher.

Copyright (c) 2022 Weinhold. This is an open-access article distributed under the terms of the Creative Commons Attribution License (CC BY). The use, distribution or reproduction in other forums is permitted, provided the original author(s) and the copyright owner(s) are credited and that the original publication in this journal is cited, in accordance with accepted academic practice. No use, distribution or reproduction is permitted which does not comply with these terms. 